\title{
An integrated circuit photocell detector and sequencer*
}

\author{
GREGORY R. GAUSTAD \\ Kansas State College of Pittsburg, Pittsburg, Kansas 66762
}

\begin{abstract}
A simple, inexpensive, and reliable apparatus for photocell detection and amplification is described. The sensitivity, voltage and current amplification, and speed of this apparatus, make it well suited to application in latency measurement in animal mazes. The apparatus can be used in any sort of application where a resistive type of transducer is needed to trigger some other event at higher voltage and current levels than can be done directly by the transducer alone.
\end{abstract}

Studies of animal learning in maze apparatus of ten use measures such as latencies or their derivatives, running speed, which depend upon the sensing of the animal's movement through the maze in an unobtrusive manner. Typically, these measuring devices involve the use of a photocell. Among the features of the photocell detector described here are: high-input impedence, enable function, reset/disable function, sequential operation, high-current output for driving solenoids or relays, single power supply operation, and simplicity of construction. The apparatus can be built for under $\$ 50$, including all parts. to the comparators has a common collector transistor configuration and therefore a very high resistance. This makes the inputs capable of detecting low-current photocell signals without appreciably influencing the signals. The outputs of the comparators are connected to a flip-flop (F-F) which provides a memory. The output transistor is capable of handling currents up to $200 \mathrm{~mA}$. The device then, serves the following functions: voltage sensing, memory, wave shaping, and current amplification. The specified range of operation is from 5 to $18 \mathrm{~V}$.

The basic photocell detector is made by forming a

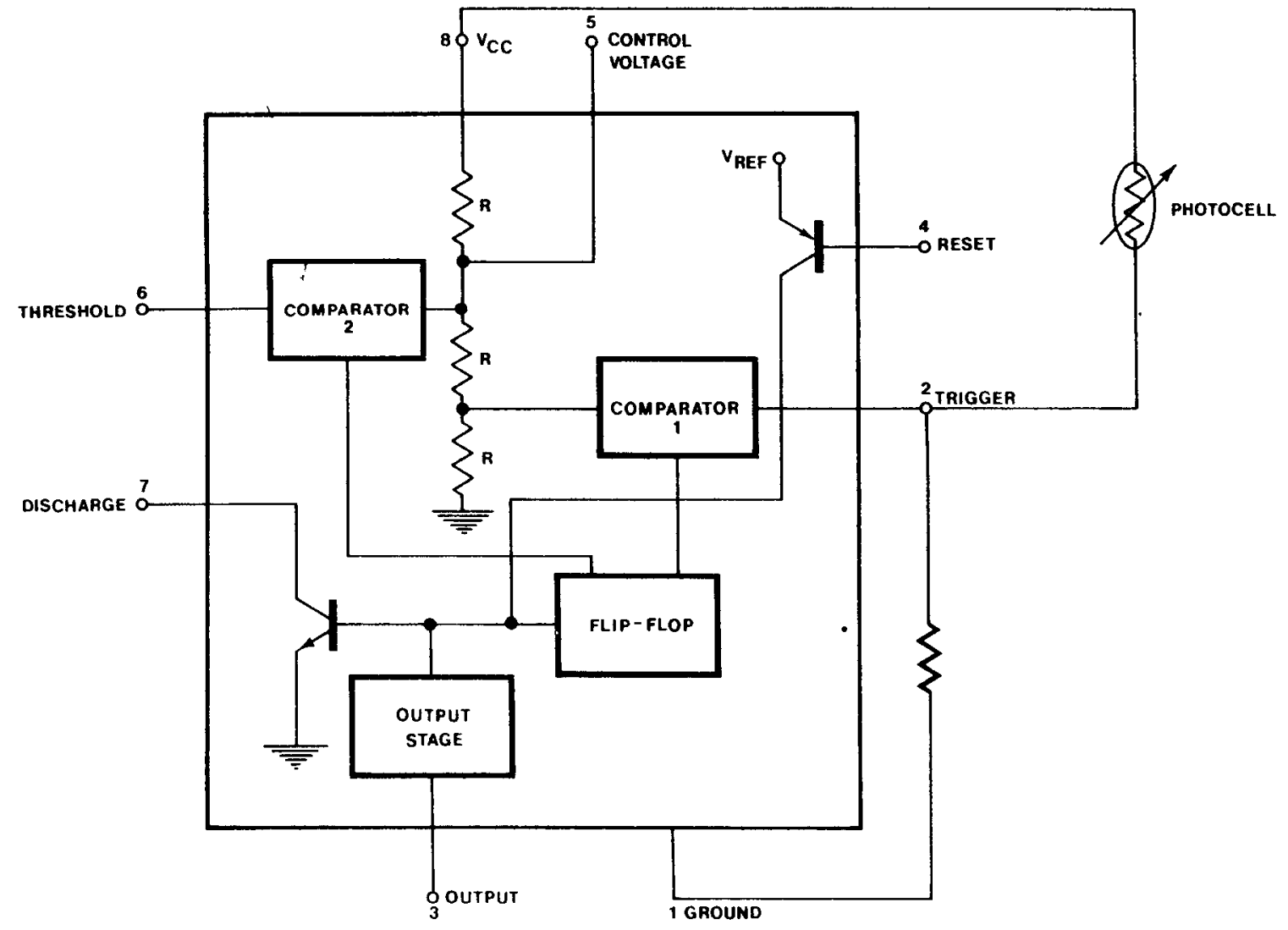

*Thanks are due to Starr Nelson for help in construction of the apparatus and also to Gary Carlat and Mitch Neymeyer for the circuit drawings.

Fig. 1. Basic photocell detector. 
The apparatus is designed so that each detector is enabled by the output of the previous detector. Therefore, one and only one detector is capable of being set (enabled) at a time. This eliminates problems due to tail blocking of previous or succeeding photocells. A pushbutton switch is used to toggle the first detector, thus enabling the second detector (usually a switch attached to the startbox door). Each successive detector is enabled and toggled in turn as the animal moves down the runway. The last detector (directly over the foodcup) toggles an adjustable $(1-60 \mathrm{sec})$ monostable multivibrator which turns on a light to indicate the time an animal should spend in the goalbox.

The heart of the apparatus is the Signetics NE555 timer integrated circuit (see Fig. 1). While the IC was designed for timing applications, it is very well suited to the present application. The inputs (Pins 2 and 6) to the NE555 are two (differential) amplifiers which function as vol tage comparators. Voltage comparators (commonly used in instruments such as digital voltmeters) are simply amplifiers that compare an unknown voltage with a reference voltage and upon equality change states very rapidly. The two voltage comparators of the NE555 are internally set for $1 / 3$ (Comparator 1 ) and $2 / 3$ (Comparator 2) of the supply voltage by a resistive voltage divider; the output of the device changes state (rise and fall times) in $100 \mathrm{nsec}$. Furthermore, the input voltage divider with a fixed resistor and a photocell at the input to one of the voltage comparators, so that when the light source is blocked from the photocell (the resistance of the photocell therefore increases) there is a drop in voltage at the input to the comparator. When the

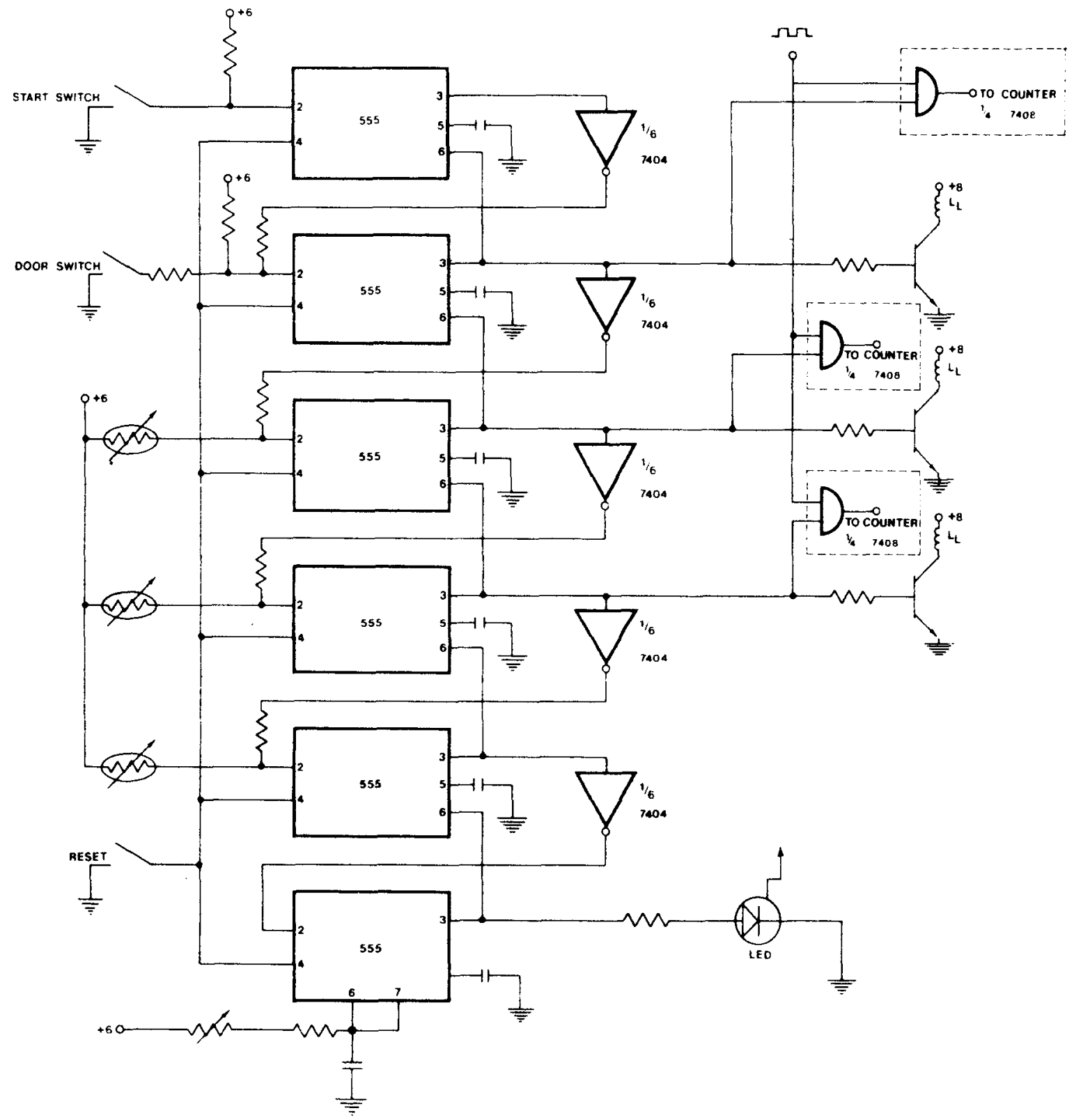

Fig. 2. The complete photocell detector. 
voltage at the input equals $1 / 3$ of the supply voltage, the comparator sets the flip-flop. The F.F will remain in the set state until reset by a positive going voltage $(2 / 3$ of the supply voltage) at the second comparator.

If the ground point of the fixed resistor is replaced with the inverted output of a previous 555 (by the Signetics 7404), then the second 555 cannot be triggered unless the previous 555 has been triggered. This is so because the input point of the second comparator is held high regardless of the resistance of the photocell (see Fig. 2). An enable function can be obtained simply by connecting a pushbutton at the first of a series of $555 \mathrm{~s}$ connected in this way. Furthermore, by connecting the output of each succeeding 555 to the reset comparator of the preceding 555 only one flip-flop can be enabled at a time, thus achieving a sequential, shift-register type of operation. The reset/disable function is achieved by connecting all of the resets of the 555 together with a toggle switch to ground. When the toggle switch is grounded, it causes the output of each 555 to be driven low. This results in the positive edge of the signal being input to the second comparator, thus causing each F.F to be reset. As long as reset is grounded, the F-Fs are disabled and cannot be activated by the photocells. As many $555 \mathrm{~s}$ as desired may be interconnected in this fashion.

The NE555 is shown in Fig. 2 in a typical application in which three measures are desired, start, run and goal latencies. This particular configuration has operated satisfactorily for thousands of trials. The outputs of the NE555 drive high current transistors that provide a small (approximately 1.1 times), but necessary, voltage gain and a large current gain. The output transistors drive Lafayette stopclocks. The Signetics 7408 AND gates (within the dashed lines) in Fig. 2 may be substituted for or used in conjunction with the transistor drivers to gate an accurate pulse train into counters. This latter method of gating a pulse train would, of course, potentially provide a much more accurate, stable, and durable measuring device, since there would be no moving mechanical parts and a highly accurate crystal controlled pulse train could be gated. Although a high resistance, slow acting photocell (Clairex CL707) was used in this application, other low resistance, faster photocells, such as the CL703L should be used in place of the CL707.

\section{REFERENCES}

Signetics Corporation. Linear data book. Sunnyvale, Calif: Signetics Corporation, 1972. Pp. 177-181.

Signetics Corporation. Digital integrated circuit $54 / 7400$. In Digital data book. Sunnyvale, Calif: Signetics Corporation, 1972. Pp. 17, 18, 25, 26.

(Received for publication December 19, 1973; revision received February 1, 1974.) 